\title{
The p.q.-Baer Property of Skew Group Rings under Finite Group Action*
}

\author{
Bo Li, Hailan Jin \\ Department of Mathematics, College of Sciences, Yanbian University, Yanji, China \\ Email: "hljin98@ybu.edu.cn, hljin98@hanmail.net
}

Received October 14, 2013; revised November 14, 2013; accepted November 20, 2013

Copyright (c) 2013 Bo Li, Hailan Jin. This is an open access article distributed under the Creative Commons Attribution License, which permits unrestricted use, distribution, and reproduction in any medium, provided the original work is properly cited.

\begin{abstract}
In this paper, Let $R$ is a ring, $G$ be a finite group of ring automorphisms of $R$. $R * G$ denote the skew group ring of $R$ under $G$. We investigate the right p.q.-Baer property of skew group rings under finite group action, Assume that $R$ is a semiprime ring with a finite group $G$ of X-outer ring automorphisms of $R$, then 1) $R * G$ is p.q.-Baer if and only if $R$ is $G$-p.q.-Baer; 2) if $R$ is p.q.-Baer, then $R * G$ is p.q.-Baer.
\end{abstract}

Keywords: p.q.-Baer Property; Skew Group Ring; Group Action

\section{Introduction}

Throughout this paper all rings are associative with identity unless otherwise stated. Let $R$ is a ring, for a nonempty subset $X$ of a ring $R, r_{R}(X)$ (resp., $l_{R}(X)$ ) denote a right (resp.,left) annihilator of $X$ in $R$. A ring $R$ is called right principally quasi-Baer (simply, right p.q.Baer) if the right annihilator of every principal right ideal of $R$ is generated, as a right ideal by an idempotent of $R$ in [1]. A left principally quasi-Baer (simply, left p.q.Baer) ring is defined similarly. Right p.q.-Baer rings have been initially studied in [1]. For more details on (right) p.q.-Baer rings, see [1-6]. A ring $R$ is called quasi-Baer if the right annihilator of every right ideal is generated, as a right ideal by an idempotent of $R$ in [7] (see also [8]. A ring $R$ is called biregular, if for each $x \in R, R x R=e R$ for some central idempotent $e \in R$. We note that the class of right p.q.-Baer rings is a generalization of classes of quasi-Baer rings and biregular rings. $Q(R)$ denote a fixed maximal right ring of quotients of $R$. Recall from [9] an idempotent $e$ of a ring $R$ is called left (resp., right) semicentral if $a e=e a e$ (resp., $e a=e a e$ ) for all $a \in R$. Equivalently, an idempotent $e$ is left (resp., right) semicentral if and only if $e R$ (resp., $\mathrm{Re}$ ) is a two-sided ideal of $R . S_{l}(R)$ (resp., $S_{r}(R)$ ) denote the set of all left (resp., right) semicentral idempotents. An idempotent $e$ of a ring $R$ is called semicentral

\footnotetext{
*Project supported by the National Natural Science Foundation of China (11361063).

${ }^{\#}$ Corresponding author.
}

reduced if $S_{l}(e R e)=\{0, e\}$. According to [2] a ring $R$ is called semicentral reduced if $S_{l}(R)=\{0,1\}$, i.e., 1 is a semicentral reduced idempotent of $R$.

If $R$ is a semiprime ring and $I$ is a two-sided ideal of $R$, then $r_{R}(I)=l_{R}(I)$. For a right $R$-module $M$ and a submodule $N$ of $M$, we use $N_{R} \leq^{\text {ess }} M_{R}$ and $N_{R} \leq{ }^{\text {den }} M_{R}$ to denote that $N_{R}$ is essential in $M_{R}$ and $N_{R}$ is dense in $M_{R}$, respectively.

Let $R$ is a ring, Aut $(R)$ denote a group of ring automorphisms of $R, G$ be a subgroup of $\operatorname{Aut}(R)$.

The skew group ring $R * G$ is defined to be

$$
R * G=\oplus \sum_{g \in G} R g
$$

with addition given component wise and multiplication given as follows: if $a, b \in R$ and $g, h \in G$, then

$$
(a b)(b h)=a b^{g^{-1}} g h \in R g h .
$$

We begin with the following example.

\section{Preliminary}

Example 2.1 There exist a ring $R$ and a finite group $G$ of ring automorphisms of $R$ such that $R$ is right p.q.-Baer but $R * G$ is not right p.q.-Baer.

Let $R=\left[\begin{array}{cc}F & F \\ 0 & F\end{array}\right]$ with a field $F$ of characteristic 2, then $R$ is right p.q.-Baer. Define $g \in \operatorname{Aut}(R)$ by

$$
g\left(\left[\begin{array}{ll}
a & b \\
0 & c
\end{array}\right]\right)=\left[\begin{array}{ll}
1 & 1 \\
0 & 1
\end{array}\right]^{-1}\left[\begin{array}{ll}
a & b \\
0 & c
\end{array}\right]\left[\begin{array}{ll}
1 & 1 \\
0 & 1
\end{array}\right] .
$$


Since characteristic of $F$ is 2, Then $g^{2}=1$.

Now we show that $R * G$ is not right p.q.-Baer. Consider the right ideal $(1+g)(R * G)$ of $R * G$ generated by $1+g$. By computation, we have

$$
\begin{aligned}
& r_{R^{*} G}((1+g)(R * G)) \\
& =\left\{\left[\begin{array}{cc}
x & y \\
0 & 0
\end{array}\right]+\left[\begin{array}{cc}
x & x+y \\
0 & 0
\end{array}\right] g \mid x, y \in F\right\}
\end{aligned}
$$

Suppose that

$$
r_{R * G}((1+g)(R * G))=e(R * G)
$$

for some $e=e^{2} \in R * G$. Note that the idempotents of $R * G$ are 0,1 .

$$
\left[\begin{array}{ll}
1 & a \\
0 & 0
\end{array}\right]+\left[\begin{array}{ll}
0 & b \\
0 & 0
\end{array}\right] g,\left[\begin{array}{ll}
0 & a \\
0 & 1
\end{array}\right]+\left[\begin{array}{ll}
0 & b \\
0 & 0
\end{array}\right] g
$$

with $a, b \in F$. Since $e \in r_{R * G}((1+g)(R * G))$, the only possible choice for $e$ is 0 . Thus if $R * G$ is right p.q.-Baer, then it follows that $r_{R * G}((1+g)(R * G))=0$. This is a contradiction. Therefore $R * G$ is not right p.q.-Baer. Also we see that $R * G$ is not left p.q.-Baer.

Definition 2.2 Let $R$ be a semiprime ring. For $g \in \operatorname{Aut}(R)$, let

$$
\phi_{g}=\left\{x \in Q_{m}(R) \mid x r^{g}=r x \text { for each } r \in R\right\},
$$

where $Q_{m}(R)$ is the Martindale right ring of quotients of $R$ (see [10] for more on $Q_{m}(R)$ ). We say that $g$ is $\mathrm{X}$-outer if $\phi_{g}=0$. A subgroup $G$ of $\operatorname{Aut}(R)$ is called $\mathrm{X}$-outer on $R$ if every $1 \neq g \in G$ is $\mathrm{X}$-outer. Assume that $R$ is a semiprime ring, then for $g \in \operatorname{Aut}(R)$, let

$$
\Phi_{g}=\left\{x \in Q(R) \mid x r^{g}=r x \text { for each } r \in R\right\} .
$$

For $g \in \operatorname{Aut}(R)$, we claim that $\Phi_{g}=\phi_{g}$. Obviously $\phi_{g} \subseteq \Phi_{g}$. Conversely, if $x \in \Phi_{g}$ then $x R=R x$. There exists $I_{R} \leq{ }^{\text {den }} R_{R}$ such that $x I \subseteq R$. Therefore $R I \triangleleft R$, $(R I)_{R} \leq^{\text {den }} R_{R}$, and $x R I=R x I \subseteq R$. Thus $x \in Q_{m}(R)$, hence $x \in \phi_{g}$. Therefore $\Phi_{g}=\phi_{g}$. So if $G$ is X-outer on $R$, then $G$ can be considered as a group of ring automorphismms of $Q(R)$ and $G$ is X-outer on $Q(R)$. For more details for $\mathrm{X}$-outer ring automorphisms of a ring, etc., see [10, p. 396] and [11].

We say that a ring $R$ has no nonzero $n$-torsion ( $n$ is a positive integer) if $n a=0$ with $a \in R$ implies $a=0$.

Lemma 2.3 Let $R$ be a semiprime ring and $G$ a group of ring automorphisms of $R$.

1) $[11,12]$ If $G$ is $X$-outer, then every nonzero twosided ideal of $R * G$ intersects $R$ nontrivially. Hence $R * G$ is semiprime.

2) [11] If $G$ is finite and $R$ has no nonzero $|G|$-torsion, Then $R * G$ is semiprime.
For a ring $R$, we use Cen $(R)$ to denote the center of $R$.

Lemma 2.4 For a semiprime ring $R$, let $G$ be a group of X-outer ring automorphisms of $R$.

Then $\operatorname{Cen}(R * G)=\operatorname{Cen}\left(R^{G}\right)$.

Proof.

Let $\alpha=a_{1} 1+a_{2} g_{2}+\cdots+a_{n} g_{n} \in \operatorname{Cen}(R)$ with $a_{i} \in R$, 1 the identity of $G$, and $g_{i} \in G$.

The

$$
\left(a_{1} 1+a_{2} g_{2}+\cdots+a_{n} g_{n}\right) b=b\left(a_{1} 1+a_{2} g_{2}+\cdots+a_{n} g_{n}\right)
$$

for all $b \in R$. So $a_{1} b=b a_{1}, a_{2} b^{g_{2}}=b a_{2}, \cdots, a_{n} b^{g_{n}}=b a_{n}$ for all $b \in R$. Since $G$ is $\mathrm{X}$-outer, it follows that $a_{2}=\cdots=a_{n}=0$. Hence $\alpha=a_{1} 1=a_{1} \in R$. Also since $\alpha b=b \alpha$ for all $b \in R$, we have that $a_{1} \in \operatorname{Cen}(R)$. Note that for all $g \in G, a_{1} g=g a_{1}=a_{1}^{g^{-1}} g$ implies $a_{1}=a_{1}^{g^{-1}}$. So $\alpha=a_{1} \in \operatorname{Cen}(R)^{G}$. Thus

$$
\operatorname{Cen}(R * G) \subseteq \operatorname{Cen}(R)^{G} \text {. }
$$

Conversely, $\operatorname{Cen}(R)^{G} \subseteq \operatorname{Cen}(R * G)$ is clear.

Therefore $\operatorname{Cen}(R * G)=\operatorname{Cen}\left(R^{G}\right)$.

Lemma $2.5[13,14]$ Let $R$ be a ring and $G$ a finite group of ring automorphisms of $R$. Then $Q(R) * G$ is the maximal right ring of quatients of $R$.

Assume that a group $G$ of ring automorphisms of a ring $R$ is finite. Then for $a \in R$, let $\operatorname{tr}(a)=\sum_{g \in G} a^{g}$, which is called the trace of $a$. Also for a right ideal $I$ of $R$, the right ideal $\operatorname{tr}(I)=\left\{\sum_{g \in G} a^{g} \mid a \in I\right\}$ of $R^{G}$ is called the trace of $I$. Say $G=\left\{g_{1}, \cdots, g_{n}\right\}$. we put $t=g_{1}+\cdots+g_{n}$ $\in R * G$. For $r \in R$ and $\alpha=a_{1} g_{1}+a_{2} g_{2}+\cdots+a_{n} g_{n}$ $\in R * G$ with $a_{i} \in R$, define $r \cdot \alpha=r^{g_{1}} a_{1}^{g_{1}}+\cdots+r^{g_{n}} a_{n}^{g_{n}}$. Then $R$ is a right $R * G$-module. Moreover, we see that ${ }_{R^{G}} R_{R^{* G}}$ is an $\left(R^{G}, R * G\right)$-bimodule.

Lemma 2.6 Assume that $R$ is a semiprime ring and $e \in B(Q(R))$. Let $I$ be a two-sided ideal of $R$ such that $I_{R} \leq^{\text {ess }} e R_{R}$ and $r_{R}(I)=f R$ with $f \in B(R)$. Then $e=1-f$.

Proof. Since $R$ is semiprime,

$$
I_{R} \leq{ }^{\text {ess }} l_{R}\left(r_{R}(I)\right)_{R}=(1-f) R .
$$

Thus

$$
I_{R}{ }^{\text {ess }}(1-f) Q(R)_{R} .
$$

As $I_{R} \leq{ }^{\text {ess }} e R_{R}, I_{R}{ }^{\text {ess }} e Q(R)_{R}$. We note that $e$ and $1-f$ are in $B(Q(R))$. So we have that $e=1-f$.

Proposition 2.7 [1] Let $R$ be a semiprime ring. Then the following are equivalent.

1) $R$ is right p.q.-Baer.

2) Every principal two-sided ideal of $R$ is right essential in a ring direct summand of $R$. 
3) Every finitely generated two-sided ideal of $R$ is right essential in a ring direct summand of $R$.

4) Every principal two-sided ideal of $R$ that is closed as a right ideal is a direct summand of $R$.

5) For every principal two-sided ideal $I$ of $R, r_{R}(I)$ is right essential in a direct summand of $R$.

6) $R$ is left p.q.-Baer.

For a ring $R$ with a group $G$ of ring automorphisms of $R$, we say that a right ideal $I$ of $R$ is $G$-invariant if $I^{g} \subseteq I$ for every $g \in G$, where $I^{g}=\left\{a^{g} \mid a \in I\right\}$. Assume that $R$ is a semiprime ring with a group $G$ of ring automorphisms of $R$. We say that $R$ is $G$-p.q.-Baer if the right annihilator of every finitely generated $G$-invariant two-sided ideal is generated by an idempotent, as a right ideal. By Proposition 2.7, if a ring $R$ is semiprime p.q.Baer with a group $G$ of ring automorphisms of $R$, then $R$ is $G$-p.q.-Baer.

A ring $R$ is called right Rickart if the right annihilator of each element is generated by an idempotent of $R$. A left Rickart ring is defined similarly. A ring $R$ is called Rickart if $R$ is both right and left Rickart. A ring $R$ is said to be reduced if $R$ has no nonzero nilpotent element. We note that reduced Rickart rings are p.q.-Baer rings.

We put

$B_{p}(Q(R))$

$=\left\{e \in B(Q(R)) \mid\right.$ there exists $x \in R$ with $\left.R x R_{R} \leq^{\text {ess }} e R_{R}\right\}$

Let $\hat{Q}_{p q B}(R)$ be the subring of $Q(R)$ generated by $R$ and $B_{p}(Q(R))$.

Lemma 2.8 [15] Assume that $R$ is a semiprime ring. Then:

1) The ring $\hat{Q}_{p q B}(R)$ is the smallest right ring of quotients of $R$ which is p.q.-Baer.

2) $R$ is p.q.-Baer if and only if $B_{p}(Q(R)) \subseteq R$.

With these preparations, in spite of Example 2.1, we have the following result for p.q.-Baer property of $R * G$ on a semiprime ring $R$ for the case when $G$ is finite and $\mathrm{X}$-outer.

\section{Main Results}

Theorem 3.1 Let $R$ be a semiprime ring with a finite group $G$ of X-outer ring automorphisms of $R$. Then $R * G$ is p.q.-Baer if and only if $R$ is $G$-p.q.-Baer.

Proof. Assume that $R * G$ is p.q.-Baer. Say

$$
I=R a_{1} R+\cdots+R a_{n} R
$$

is a finitely generated $G$-invariant two-sided ideal of $R$ with $a_{i} \in R$. Then $I * G$ is a two-sided ideal of $R * G$. Moreover,

$$
I * G=(R * G) a_{1}(R * G)+\cdots+(R * G) a_{n}(R * G),
$$

Note that $R * G$ is semiprime by Lemma2.3, So Propo- sition 2.7 yields that there exists $e \in S_{l}(R * G)$ such that

$$
(I * G)_{R * G} \leq^{\text {ess }} e(R * G)_{R * G} .
$$

Since $R * G$ is semiprime, $e \in B(R * G)$ by [9]. Hence by Lemma2.4, $e \in \operatorname{Cen}(R)^{G}$. First, we see that $I_{R} \leq^{\text {ess }} e R_{R}$. For this, let $0 \neq e r \in e R$ with $r \in R$. As $(I * G)_{R * G} \leq^{\text {ess }} e(R * G)_{R * G}$, there exists $\beta \in R * G$ such that $0 \neq e r \beta \in I * G$.

Say $\beta=b_{1} g_{1}+\cdots+b_{n} g_{n}$ with $b_{i} \in R$ and $g_{i} \in G$ for $i=1, \cdots, n$. Then

$$
\operatorname{er} \beta=\left(e r b_{1}\right) g_{1}+\cdots+\left(e r b_{n}\right) g_{n} \in I * G .
$$

Hence $0 \neq e r b_{j} \in I$ for some $j$, so $I_{R} \leq^{\text {ess }} e R_{R}$. As $e=e^{2} \in \operatorname{Cen}(R)^{G}, I \subseteq e R e$, and so $I_{e R e} \leq^{e s s} e R e_{e R e}$.

Now we show that $r_{R}(I)=(1-e) R$. If $e=0$, then $r_{R}(I)=R$. So we may assume that $e \neq 0$. Note that $e R e$ is semiprime and $I_{e R e} \leq^{\text {ess }} e R e_{e R e}$, and so $r_{e R e}(I)=0$. Hence

$$
e R \bigcap r_{R}(I)=e R e \bigcap r_{R}(I)=0 .
$$

As $I \subseteq e R,(1-e) R \subseteq r_{R}(I)$. From the modular law,

$$
r_{R}(I)=(1-e) R \oplus\left(e R \bigcap r_{R}(I)\right) .
$$

But since $e R \cap r_{R}(I)=0, r_{R}(I)=(1-e) R$. Therefore $R$ is $G$-p.q.-Baer.

Conversely, let $R$ be $G$-p.q.-Baer. Take

$$
e \in B_{p}(Q(R) * G) .
$$

Then

$$
e \in[\operatorname{Cen}(Q(R))]^{G}
$$

by Lemma 2.4 since $G$ is also X-outer on $Q(R)$ as was noted. Also there exists $\alpha \in R * G$ such that

$$
(R * G) \alpha(R * G)_{R * G} \leq^{\text {ess }} e(R * G)_{R * G}
$$

because $Q(R) * G$ is the maximal right ring of quotients of $R * G$ (Lemma 2.5) and $e \in B_{p}(Q(R) * G)$. Say $\alpha=a_{1} g_{1}+a_{2} g_{2}+\cdots+a_{n} g_{n}$ with $a_{i} \in R$ and $g_{i} \in G$ for $i=1,2, \cdots, n$. Then $\alpha \in e(R * G)(e R) * G$ and so $a_{i} \in e R$ for each $i=1,2, \cdots, n$. Consider $K=\sum_{i=1, g \in G}^{n} R a_{i}^{g} R$. Then $K$ is a finitely generated $G$-invariant two-sided ideal of $R$. Further, $K \subseteq e R$ because $e \in[\operatorname{Cen}(Q(R))]^{G}$. By the preceding argument, we see that $K_{R} \leq^{\text {ess }} e R_{R}$. From the assumption, there exists $f \in S_{l}(R)=B(R)$ such that $r_{R}(K)=f R$. Thus $e=1-f \in R$ by Lemma 2.6. Therefore $e \in R \subseteq R * G$, so $B_{p}(Q(R) * G) \subseteq R * G$. From 
Lemma2.8, $R * G$ is p.q.-Baer.

Corollary 3.2 Let $R$ be a semiprime ring with a finite group $G$ of X-outer ring automorphisms of $R$. If $R$ is p.q.-Baer, then $R * G$ is p.q.-Baer.

Proof. The proof follows immediately by Theorem 3.1 .

\section{Conclusion}

In [16] researched quasi-Baer property of skew group rings under finite group actions on a semiprime ring and their applications to $C^{*}$-algebras (see also $[17,18]$ ). In this paper, we investigate the right p.q.-Baer property of skew group rings under finite group action. Assume that $R$ is a semiprime ring with a finite group $G$ of $\mathrm{X}$-outer ring automorphisms of $R$, then 1$) R * G$ is p.q.-Baer if and only if $R$ is $G$-p.q. -Baer; 2) if $R$ is p.q.-Baer, then $R * G$ is p.q.-Baer.

\section{REFERENCES}

[1] G. F. Birkenmeier, J. Y. Kim and J. K. Park, "Principally Quasi-Baer Rings,” Communications in Algebra, Vol. 29, No. 2, 2001, pp. 639-660. http://dx.doi.org/10.1081/AGB-100001530

[2] G. F. Birkenmeier, H. E. Heatherly, J. Y. Kim and J. K. Park, "Triangular Matrix Representations," Journal of Algebra, Vol. 230, No. 2, 2000, pp. 558-595. http://dx.doi.org/10.1006/jabr.2000.8328

[3] G. F. Birkenmeier, J. Y. Kim and J. K. Park, “Quasi-Baer Ring Extensions and Biregular Rings," Bulletin of the Australian Mathematical Society, Vol. 61, No. 1, pp. 3952.

[4] G. F. Birkenmeier, J. Y. Kim and J. K. Park, "A Sheaf Representation of Quasi-Baer Rings," Journal of Pure and Applied Algebra, Vol. 146, No. 3, 2000, pp. 209-223. http://dx.doi.org/10.1016/S0022-4049(99)00164-4

[5] G. F. Birkenmeier and J. K. Park, "Triangular Matrix Representations of Ring Extensions," Journal of Algebra, Vol. 265, No. 2, 2003, pp. 457-477.

http://dx.doi.org/10.1016/S0021-8693(03)00155-8

[6] G. F. Birkenmeier, J. Y. Kim and J. K. Park, "Polynomial Extensions of Baer and Quasi-Baer Rings,” Journal of Pure and Applied, Vol. 159, No. 1, 2001, pp. 25-42. http://dx.doi.org/10.1016/S0022-4049(00)00055-4
[7] W. E. Clark, "Twisted Matrix Units Semigroup Algebras,” Duke Mathematical Journal, Vol. 34, No. 3, 1967, pp. 417-423. http://dx.doi.org/10.1215/S0012-7094-67-03446-1

[8] A. Pollingher and A. Zaks, "On Baer and Quasi-Baer Rings,” Duke Mathematical Journal, Vol. 37, No. 1, 1970, pp. 127-138. http://dx.doi.org/10.1215/S0012-7094-70-03718-X

[9] G. F. Birkenmeier, "Idempotents and Completely Semiprime Ideals," Communications in Algebra, Vol. 11, No. 6, 1983, pp. 567-580. http://dx.doi.org/10.1080/00927878308822865

[10] T. Y. Lam, "Lectures on Modules and Rings," Springer, Berlin, 1998.

[11] J. W. Fisher and S. Montgomery, "Semiprime Skew Group Rings,” Journal of Algebra, Vol. 52, No. 1, 1978, pp. 241-247. http://dx.doi.org/10.1016/0021-8693(78)90272-7

[12] S. Montgomery, "Outer Automorphisms of Semi-Prime Rings,” Journal London Mathematical Society, Vol. 18, No. 2, 1978, pp. 209-220. http://dx.doi.org/10.1112/jlms/s2-18.2.209

[13] K. Louden, "Maximal Quotient Rings of Ring Extensions," Pacific Journal of Mathematics, Vol. 62, 1976, pp. 489496.

[14] J. Osterburg and J. K. Park, "Morita Contexts and Quotient Rings of Fixed Rings," Houston Journal of Mathematics, Vol. 10, 1984, pp. 75-80.

[15] G. F. Birkenmeier, J. K. Park and S. T. Rizvi, "Principally Quasi-Baer Ring Hulls: in Advances in Ring Theory,” In: D. V. Huynh and S. R. López-Permouth, Tends in Math., Birkhauser, Boston, 2010, pp. 47-61. http://dx.doi.org/10.1007/978-3-0346-0286-0 4

[16] H. L. Jin, J. Doh and J. K. Park, "Group Actions on Quasi-Baer Rings," Canadian Mathematical Bulletin, Vol. 52, 2009, pp. 564-582. http://dx.doi.org/10.4153/CMB-2009-057-6

[17] G. F. Birkenmeier, J. K. Park and S. T. Rizvi, "Hulls of Semiprime Rings with Applications to $C^{*}$ Algebras," Journal of Algebra, Vol. 322, No. 2, 2009, pp. 327-352. http://dx.doi.org/10.1016/j.jalgebra.2009.03.036

[18] G. F. Birkenmeier, J. K. Park and S. T. Rizvi, "The Structure of Rings of Quotients,” Journal of Algebra, Vol. 321, No. 9, 2009, pp. 2545-2566. http://dx.doi.org/10.1016/j.jalgebra.2009.02.013 